\title{
Correction to: "Reality" and Representation in Mechanics: The Legacy of Walter Noll
}

\author{
Gianpietro Del Piero, ${ }^{1,2}$
}

Published online: 20 September 2019

(C) Springer Nature B.V. 2019

\section{Correction to: J. Elast. (2019) 135: 117-148 https://doi.org/10.1007/s10659-018-9697-y}

This erratum concerns a series of misprints, due to a human error occurred after the final proofreading. Due to this error, some capital $F$ of the manuscript became lower-case $f$ in the final version. Precisely, all $f$ contained in equations (37) to (40) and (78), (79), (85), (90) should be read as $F$. For the readers' convenience, below I transcribe the corrected forms:

$$
\begin{aligned}
& \mathscr{E}_{i n t}\left(\Omega_{R}\right)=\mathscr{E}_{\text {int }}\left(\Omega_{R}, F, F^{d}, \nabla F, \nabla F^{d}\right), \\
& \mathscr{E}_{\text {int }}\left(\Omega_{R}, F, F^{d}, \nabla F, \nabla F^{d}\right)=\int_{\Omega_{R}}\left(\varphi(F, \nabla F)+\varphi^{d}\left(F^{d}, \nabla F^{d}\right)+\phi^{d}\left(F^{d}, \nabla F^{d}\right)\right) \mathrm{dv}, \\
& \mathscr{P}_{\text {int }}\left(\Omega_{R}, V, V^{d}, \nabla V, \nabla V^{d}\right)=\int_{\Omega_{R}}\left(\nabla_{1} \varphi(F, \nabla F) \cdot V+\nabla_{2} \varphi(F, \nabla F) \cdot \nabla V\right. \\
& +\nabla_{1} \varphi^{d}\left(F^{d}, \nabla F^{d}\right) \cdot V^{d}+\nabla_{2} \varphi^{d}\left(F^{d}, \nabla F^{d}\right) \cdot \nabla V^{d} \\
& \left.\quad+\breve{\nabla}_{1} \phi^{d}\left(F^{d}, \nabla F^{d}\right) \triangleright V^{d}+\breve{\nabla}_{2} \phi^{d}\left(F^{d}, \nabla F^{d}\right) \triangleright \nabla V^{d}\right) \mathrm{dv}, \\
& \breve{\nabla}_{1} \phi^{d}\left(F^{d}, \nabla F^{d}\right) \triangleright V^{d}+\breve{\nabla}_{2} \phi^{d}\left(F^{d}, \nabla F^{d}\right) \triangleright \nabla V^{d}>0,
\end{aligned}
$$

The online version of the original article can be found under https://doi.org/10.1007/s10659-018-9697-y.

\footnotetext{
G. Del Piero

dlpgpt@unife.it

1 Dipartimento di Ingegneria, Università di Ferrara, 44100 Ferrara, Italy

2 International Research Center M\&MoCS, Cisterna di Latina, Italy
} 


$$
\begin{aligned}
& Y \cdot \int_{\Pi_{R}}\left(T_{R} \nabla f^{T}+\left(\operatorname{div} \mathbb{T}_{R}+B_{R}\right) F^{T}+\mathbb{T}_{R} \nabla F^{T}\right) \mathrm{d} v=0, \\
& \left(T_{R} \nabla f^{T}+\left(\operatorname{div} \mathbb{T}_{R}+B_{R}\right) F^{T}+\mathbb{T}_{R} \nabla F^{T}\right)^{W}=0,
\end{aligned}
$$

Moreover, the correct forms of (85) and (90) are

$$
\begin{aligned}
& \left(T_{R} \nabla f^{T}+\mathbb{T}_{R} \nabla F^{T}\right)^{W}=0, \\
& \psi=\psi\left(F, \nabla F^{d}\right) .
\end{aligned}
$$

For the sentence between (37) and (38) reported below, the correct form is:

"The subsequent extension to second-order structured deformations, to be identified with two-scale deformations, led to the additional dependence on $\nabla F$. Here, the dependence on $\nabla F^{d}$ has been added."

The text between (39) and (40),

"is obtained, with $\nabla_{1}, \nabla_{2}$ partial derivatives with respect to $F, F^{d}$ and $\nabla F, \nabla F^{d}$, with $V, V^{d}$ virtual velocities for $F$ and $F^{d}$, and with".

Moreover, at the beginning of Sect. 6.4,

"For a classical continuum, the energy densities $\varphi, \varphi^{d}$ are assumed to be independent of $\nabla F$ and $\nabla F^{d}$, respectively.",

at the beginning of Sect. 7.3,

"Then setting $\nabla v=V+V^{d}$ and omitting the arguments $(F, \nabla F),\left(F^{d}, \nabla F^{d}\right)$, we get..."

and in Footnote 48,

"In components, $\left(\operatorname{div} \mathbb{T}_{R} F^{d T}\right)_{i h}=\mathbb{T}_{i j k, k} F_{h j}^{d}$ and $\left(\mathbb{T}_{R} \nabla F^{d T}\right)_{i h}=\mathbb{T}_{i j k} F_{h j, k}^{d} "$

Finally, the sentence between (83) and (84) should be read as:

"Moreover, taking $\varphi^{d}$ and $\phi^{d}$ independent of $\nabla F^{d}$ in accordance with [3], the activation condition for $\delta F^{d}$ reduces to ...".

Publisher's Note Springer Nature remains neutral with regard to jurisdictional claims in published maps and institutional affiliations. 